\title{
Evaluation of Hospital Laboratory Workflow Design in Ethiopia: Blood Specimen Collection and Chemistry
}

\section{Laboratory Testing}

\section{Eshetu LH* \\ Africa Quality Consulting Firm PLC, Ethiopia}

*Corresponding author: Eshetu Lemma Haile, Africa Quality Consulting Firm PLC, Addis Ababa, P.O.Box. 21790 codes 1000, Ethiopia, Email: eshetulema@gmail.com

\section{Research Article \\ Volume 2 Issue 5}

Received Date: August 19, 2018

Published Date: September 17, 2018

DOI: $10.23880 /$ eoij-16000179

\section{Abstract}

Background: Laboratories have recognized their internal business and operate as a set of business processes or workflows. Modern Laboratories are highly suitable to this workflow driven approach. Evaluation of current workflow and re-design processes are necessary and requirements of customers to increase customer needs and efficiency in daily laboratory operation. In Ethiopia there is no baseline information on hospital laboratory workflows sited on literature.

Objective: To evaluate existed workflow in clinical hospital laboratory and proposed new workflow in Addis Ababa, Ethiopia.

Method: A cross-sectional study design was used. Quantitative method and direct observation were conducted. Standardized checklist and questionnaire was used to assess the workflow of existed automated chemistry laboratory testing and OPD specimen collection workflow. To assessed these the study drawn a map of the sample`s journey using a tool called value stream mapping; spaghetti diagram and record detail of workflow. A new workflow simulation model is proposed based on adoption of Vacutainer blood collection device and its associated tubes instead of syringe needle.

Results: The average time of samples waiting at reception after collection and prior to delivery to respective laboratories were decline as more samples collected within short time interval. The average cycle times of 489 blood sample drawing processes were 3.58 minutes and 76.5\% (374) of blood samples were drawn with an average cycle time of 1.0 to 5.0 minutes. A total of 1231.31 minutes were identified as non-value added (waste) time from existed workflow of chemistry laboratory testing process and seven non-valued added process steps were recognized. Clinical chemistry laboratory process cycle efficiency was $56.6 \%$. New simulation workflow is redesign by considering Vacutainer blood collection device and their associated tubes are being used rather than syringe needle. Accordingly simulated specimen collection workflow were reduced to 19 steps from existed workflow of 28 steps, 18 major steps were proposed for new chemistry 


\section{Ergonomics International Journal}

laboratory testing workflow from 26 steps; and the movement of operator could be reduced significantly into six steps as compared 17 steps of movement where 12 operator`s movements were considered as wastes.

Conclusion: Laboratories should identified non-value added process, steps or activities and by eliminating those wastes and implementing a powerful business improvement tools like lean method possible to maximized and redesign process to meet customer needs. Laboratories should assess their workflow periodically to identify wasteful practices and focused only value-added activity to increase the process cycle efficiency and productivity.

Keywords: Workflow; process; Lean; laboratory, specimen collection; hospital, Ethiopia, design, Lead time; cycle efficiency, value-added; non-value added; waste; value stream mapping; Spaghetti diagram; movement.

\section{Introduction}

Lean is problem solving tool and derived from Japanese automobile industry. It's a systematic approach to improving the reliability of processes through identification and elimination of operational barriers and sources of variability within a process or system [1].

Leans Systems Approach (LSA) improves process by identifying each step, removing non-value added steps, and eliminating waste. The benefits of applying such principles will make a more efficient, timely delivery of products and meeting customer needs [2]. Laboratories can adopt lean principles (after careful work flow and work load evaluation) that can decrease unnecessary steps [3].

The impact of applying lean management principles to specimen collection and handling process shown that Non-value-added or error-prone steps are either removed or controlled in the revised process, resulting in a more efficient, timely, and standardized process. Specifically, by changing the method of specimen collection, unnecessary steps could be removed from the process. Some of errorprone steps could be eliminated; some may be from preanalytical phase, example the open transfer of specimens into tubes from cups and the relabeling of specimens and thus possible to increase the efficiency and reduced the waste of the laboratory staff and materials [4]. Furthermore, a well design laboratory with efficient workflow is paramount in producing timely, accurate and reliable result, and safety of laboratory personnel's [5]. Therefore, redesigning of workflow whenever necessary could be considered as a way to remove non-value steps and increase laboratory efficiency.

\section{Methods}

A cross-sectional study design was conducted to evaluate workflow efficiency at selected hospital laboratory in Addis Ababa. Quantitative methods and direct observation were used. Standardized checklist and questionnaire were used to assess the workflow of existed automated chemistry laboratory testing process and OPD blood specimen collection workflow. To assessed these the study drawn a map of the sample`s journey using a tool called value stream mapping; spaghetti diagram and record detail of workflow. The objective of this study was to evaluate the existed laboratory specimen collection and chemistry laboratory testing workflow and propose new workflows.

Moreover, the team took photographs and video of current workflow and motion of work. Using photographs and video, the team had a chance to realize how work and process was setup and able to identified non-value added steps and identified gaps that could be improve. Based on this, the team established a more efficient redesign workflow and compared with existed workflows.

Clinical chemistry laboratory unit was selected based on large number of test volume analyzed in the previous year. Key tools include: value stream mapping (capture details of process), spaghetti diagrams (map motion); measure times (Cycle time-from beginning to completion and lead time) was used in this study.

The project took 4 weeks for purpose of reassuring measure and data to avoid day variation and conditions. During this period the group was come up with existed detail workflow and identified areas that need changes 


\section{Ergonomics International Journal}

which would be used for developing a new proposed workflow. The ethical committee of Addis Ababa University Medical Faculty, Institutional Review Board (IRB) was approved the study. Permission was secured from selected hospital.

\section{Chemistry Laboratory Testing and Sample Collection Process: Data Capturing Steps}

Step-1: Data was collected based on pre-checked checklist on pre-analytical, analytical, and post analytical phases.

Step-2: Data was collected through interview of lab staff about workflow and consulting with process documentation.

Step-3: Detail observational data was collected on workflow process and record the whole activities every day using photograph, video and paper.

Step-4: The existing workflow motion was drawn by spaghetti tool.

Step-5: Each and every single chemistry laboratory testing activities against time were captured by value stream mapping.

Step-6: Identified areas that need improvement and cut any wasteful work processes.

Step-7: Developed new ideal proposed workflow for chemistry lab testing process and blood sample collection workflow and

Step-8: New spaghetti workflow motion and value stream mapping was drawn.

\section{Operational Definition}

- Lean Systems Approach (LSA): Improves process by identifying each step, removing non-value added steps, and eliminating waste.

- Non-value add time: anything that doesn't add value is waste such as unnecessary activities like long specimen transportation time, waiting time, processing time, motion time, spending time on finding tools or equipment, rework time, etc

- Process Cycle Efficiency: Value Add Time/Total Lead Time.

- Process: A series of steps or actions that produces a completed order or product.

- Value stream map: graphical depiction of the sequence of processes for a product and key characteristics of each process.
- Value-add time: work that a customer would recognize as necessary to create the product or service they are about to purchase.

- Workflow: the pathway the work follows from the beginning to the end of task.

\section{Results}

Workflow analysis was conducted to assess two main processes of laboratory: specimen collection and chemistry laboratory testing workflow.

\section{Specimen Collection Workflow}

A total of 708 samples were followed, of these 489 were blood chemistry samples. Every day with range of 20 to 25 samples were followed for consecutive 30 working days. The detail existed steps is depicted in figure 1 and the new simulated proposed specimen collection workflow is redesigned by changing syringe needle device into Vacutainer blood collection techniques and use respective tubes (Figure 2). There were a total of 28 existed major steps starting from test order until delivery of specimen to various departments (Figure 1). In figure 2 shown that the simulated workflow were reduced into 19 steps compared from existed workflow which had 28 steps. The modified workflow could save much time by shorting the workflow which had no value for customers and operators. The existed blood specimen collection process was used syringe needle system and had a process of aliquoting specimen into different tubes.

Workflow evaluation was done for outpatient population who had started their journey by visiting doctor office for examination and test orders. Then patient walked into result distribution office for purpose of receiving payment amount/quotation for test being render and then went to casher officer for payment and again went back to same office for payment confirmation. Patient waited at waiting place until their queue reached and the coordinator sorted out patient based on sample types and first handover patient who had stool and urine test orders and then to blood collection processes.

On average each patient had to wait 40 seconds at the result distribution. The patient also waited on average 2.21 minutes at payment office and 6 seconds for verification purpose. Similarly, a patient should be waited averagely 1.50 minutes at the blood drawing station, and 2.35 minutes were recorded averagely for drawing blood 
process per patient. Blood collected for hematological tests were left on the mixer for maximum and minimum of 1.05 hour and 2.3 minutes respectively and on average after initial hand mixing the blood left further mixing for 23.32 minutes. A sample was stayed at reception in range of 13.0 minutes to 1.15 hours. On average transcribing hospital ID number into logbook was 14 seconds per patient form.

To complete the whole process of sample collection workflow for 708 samples, it required a total of 1061.12 minutes. A total of 649.2 minutes were identified as nonvalue added time from 708 (489 blood samples and 219 other sample). In this workflow non-added value steps were identified and the major were time of patient visit result distribution office and identify the amount of money to be paid per test, patient comeback to the result distribution room for payment confirmation, writing down hospital ID number into worksheet, time spent on delivery of samples, etc.

The major factor for non-value added time were leaving the haematology blood sample for more than 23.32 minute in average, time spent on transportation of sample, writing the hospital ID number into worksheets and collecting and transporting the paediatric samples into OPD reception.

Figure 3 illustrated that after the blood samples drawn the average time of waiting at reception prior to delivery were decreased as more samples collected within short time interval. If small number of samples, one to ten, collected blood samples could be waited more time at reception before delivery to laboratory due to batch system is used.

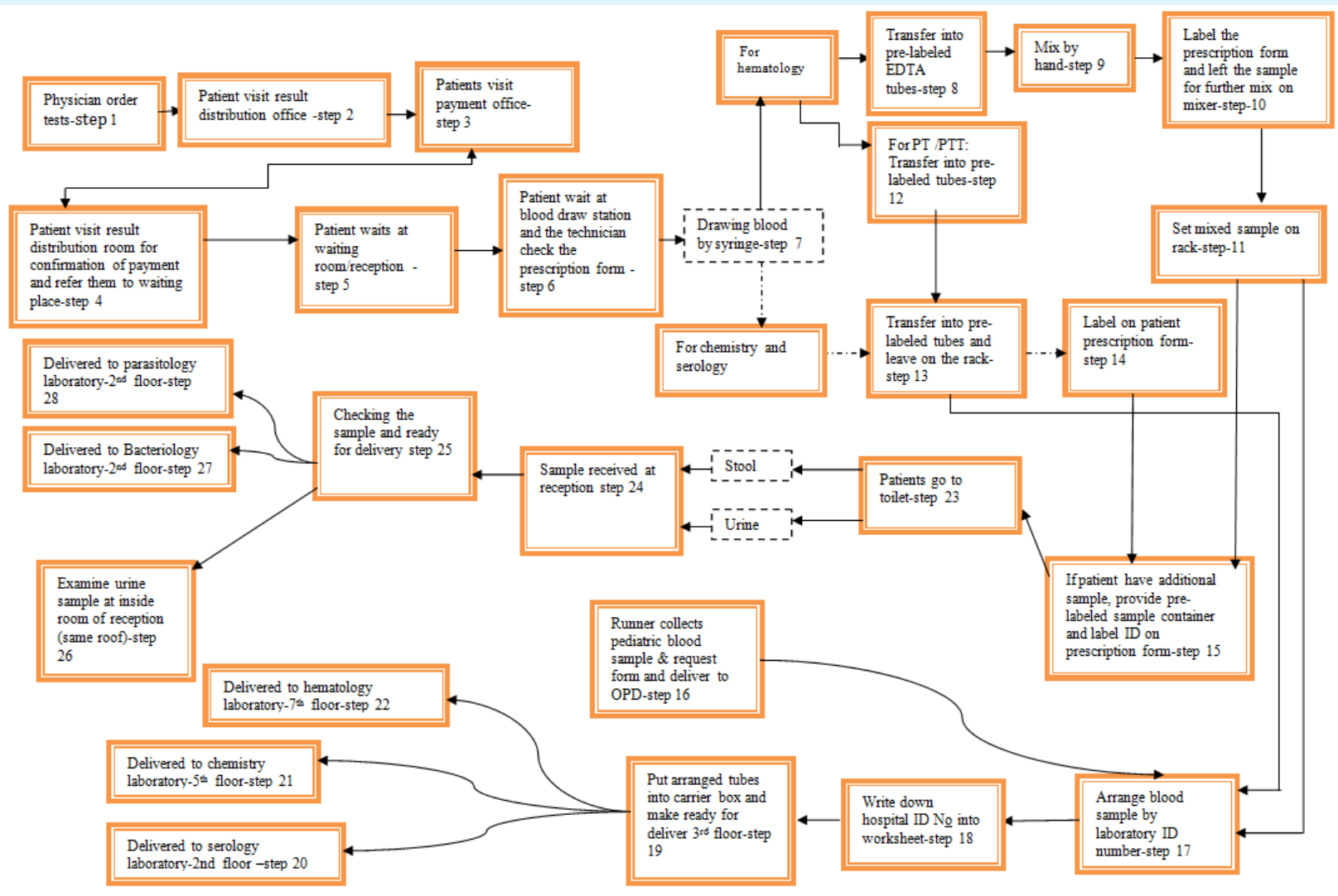

Figure 1: Specimen Collection Workflow-“As Is", Hospital, Addis Ababa, Ethiopia. 


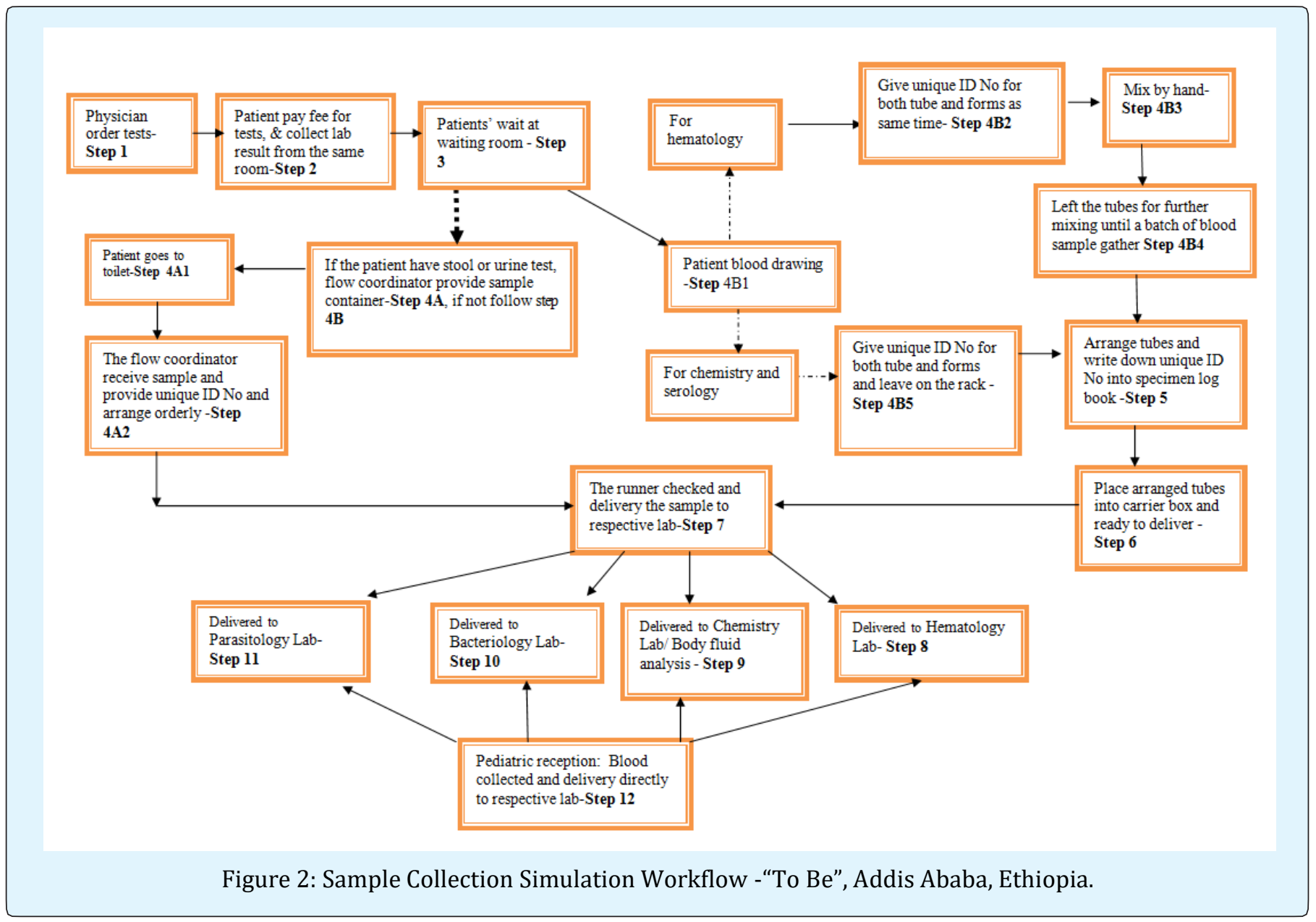

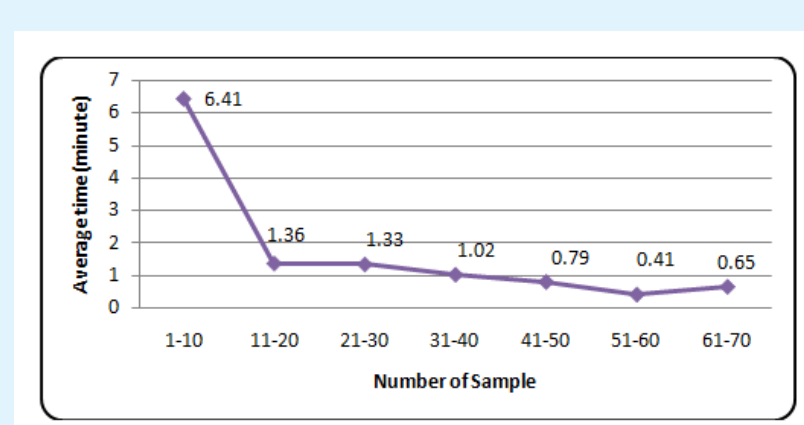

Figure 3: Average Time Of Patient Samples Waited At Reception After Blood Drew (Per Minutes), Addis Ababa, Ethiopia.
The average cycle time of blood draw process were defined as the time between the patients presented and seated at the blood drawing chair and end time of blood draw. The average cycle times for 489 blood drawing process were 3.58 minutes and $76.5 \%$ (374) of patients were provided their blood with an average cycle time of 1.0 to 5.0 minutes (Figure 4).

Moreover, the study found that on average 1.15 minutes spent on blood drawing cycle time were used for 15 patients as compared to 1 patient that was 2.54 minutes. Besides that 90 th percentile of the cycle time and 50th of the cycle time were below 6.41 and 3.39 minutes respectively. 


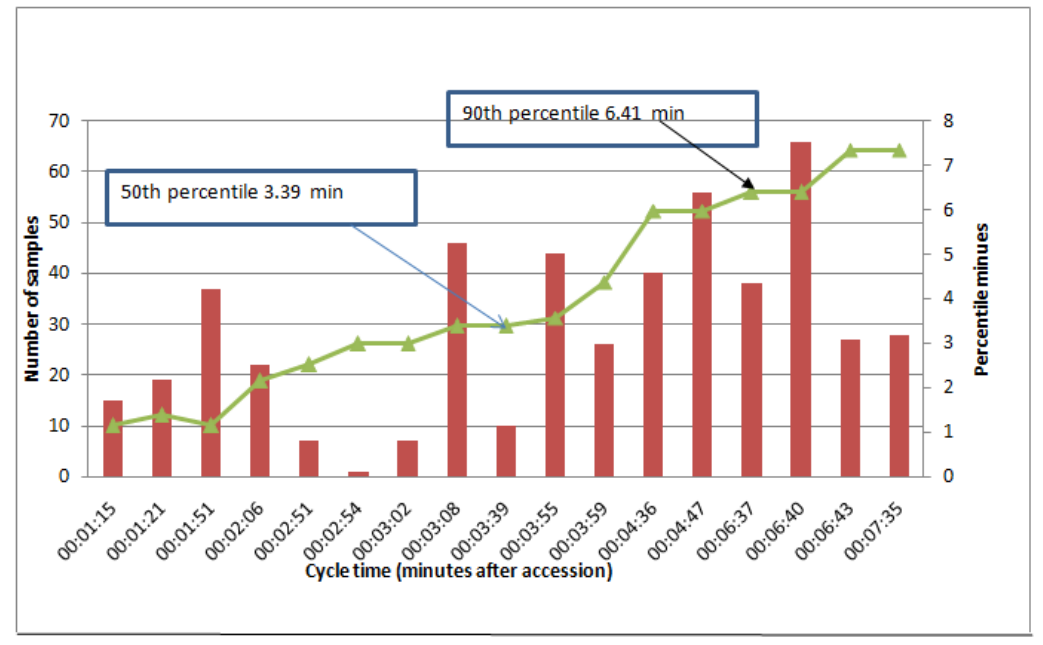

Figure 4: Average CYCLES time of a patient blood draw process (minutes after accessioning), Addis Ababa, Ethiopia.

Figure 5 shows that on average one-way delivery of blood samples from reception to serology, chemistry and haematology laboratory section were $2.54,3.39$ and 4.3 minutes respectively. A total of 21.14 minutes (1032 meters) were spent on transportation of sample per one deliver into five laboratory sections.
When sample collection workflow analyzed for chemistry blood samples only excluding the other sample collection workflow, a total of 443.42 minutes were recorded as non-value added time out of 833.19 minutes.

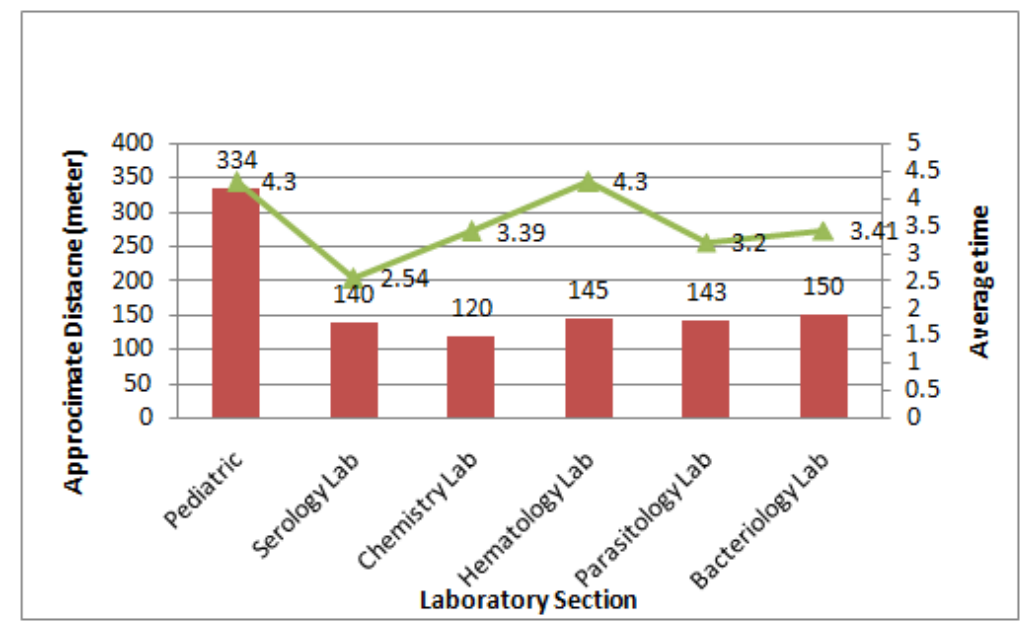

Figure 5: Distance Vs Average time from reception to laboratory sections, Addis Ababa, Ethiopia.

The study was also investigate reception opening hours and actual working hours focusing on patient who couldn't able to give their specimen due to shortage of time in previous day. Accordingly, the average patient 


\section{Ergonomics International Journal}

waiting time before opening of reception was 6.06 minutes and the minimum and maximum time of waiting was 2.1 and 14.56 minutes. Most patients waited after opening of reception and prior to present blood drawing station were in range between 2 and 6.0 minutes. Similarly most patients were arrived much early than official opening time of reception and averagely the patient should wait 1.21 hour to get service and with range of 35 minutes (Minimum) to 2.28 hours (Maximum). Only 41.2\% (201/489) of samples were got a chance to have the service less than one hour.

\section{Chemistry Laboratory Testing Workflow}

A total of 489 chemistry blood samples were followed and the minimum number of chemistry blood sample followed was one and the maximum was 16 samples per day. On average a sample could took a total of 15:12 minutes compared with aggregated samples which took 6:34 hours start to end (Figure 6). There were 26 major process steps identified with existed workflow. The new simulated proposed chemistry laboratory testing workflow is reduced into 18 major processes (Figure 7). Note that the new workflow was not evaluated against actual time.

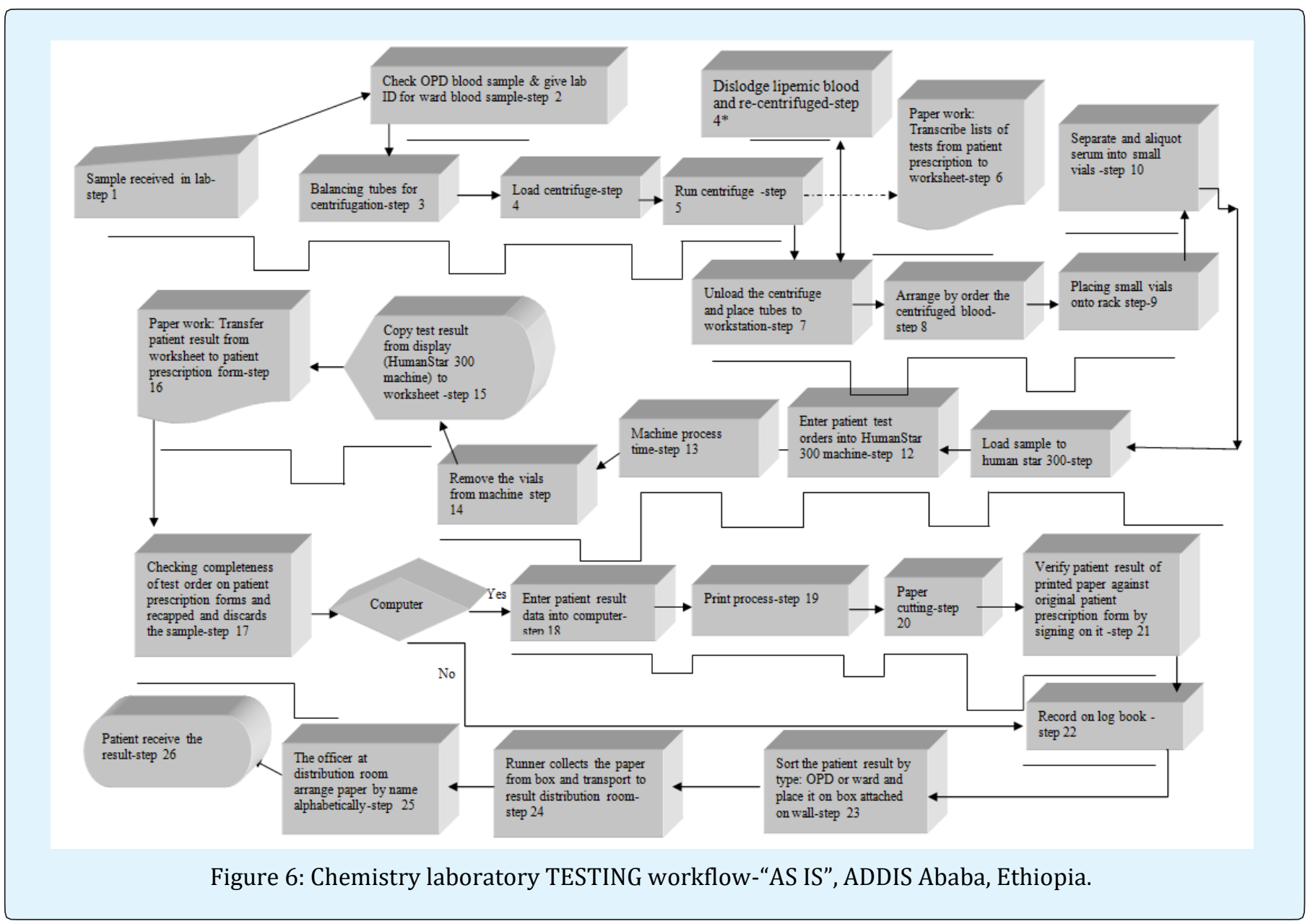

Eshetu LH. Evaluation of Hospital Laboratory Workflow Design in Ethiopia: 


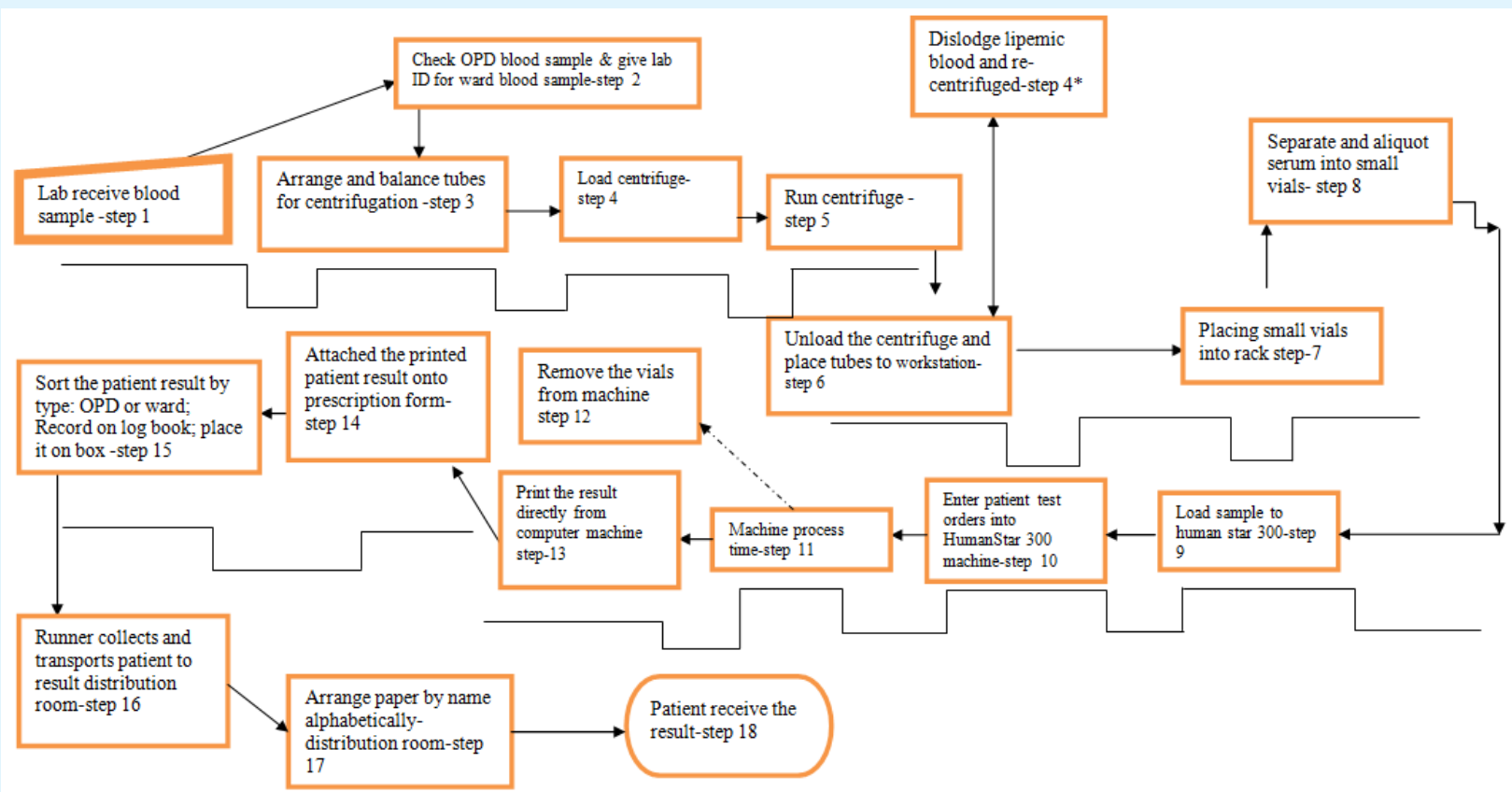

Figure 7: Chemistry Laboratory Testing Simulated Workflow-“To Be”, Addis Ababa, Ethiopia.

A total time to complete 489 chemistry blood samples after received, analyzed and made result ready for patient were 3025.67 minutes (50.42 hours). Excluding the machine hour (analytical phases), a total of 1771.26 minutes (29.52 hours) were spent on post-analytical phase such as copy test result from display (HumanStar 300 machine) to worksheet; transferring the patient result from worksheet to patient prescription form; enter patient result data into computer or record on log book; print process and paper cutting; verification of patient results, etc and a total of 641.43 minutes (11.09 hours) were utilized by pre-analytical phases such as load centrifuge; arrange centrifuged blood by order; separate and aliquot serum into vials; unload the centrifuge and place tubes to workstation. Therefore, comparatively $58.5 \%$ of times were spent on post-analytical phases as compare to $21.2 \%$ of pre-analytical phases. The machine hours were relatively smaller by 613.38 (10.21 hours) minutes compared to pre- and post-analytical phases.

The workflow of chemistry blood samples test, a total of 1231.31 minutes were identified as non-value added (waste) time from existed workflow and seven nonvalued added process steps recognized. This can be explained in terms of unit sample that was 2.51 minutes (1231.31/489) added in each sample which had no value in customer point of view. From total non-added value, $86.7 \%$ could explained on post-analytical phases such as copy test result from display (HumanStar 300 machine) to worksheet, transfer patient result from worksheet to patient prescription form, enter patient result data into computer, and verify patient result on printed paper against original patient prescription form by signing on it and paper cutting. The rest (13.3\%) non-valued added time were identified on pre-analytical phases such as transcribe lists of tests from patient prescription to worksheet, arrange the centrifuged by blood order.

\section{Chemistry Blood Test: Cycle and Lead Time}

The average cycle time of chemistry blood test is defined as the time of specimen received at the chemistry laboratory and time where the test result ready for runner to be taken. The average cycle time was found 2.46 hours when computerized system was used, however, for manual process the average cycle time were 2.19 hours.

The blood chemistry lead time were defined as the time where the patient arrive at reception to give blood samples to time where the chemistry laboratory results ready for the patient. The lead time were defined contextually for this study. 
The total lead times were 3858.19 minutes and total value-added times for chemistry blood process were 2184.13 minutes. Therefore, the chemistry process cycle efficiency was $56.6 \%$. In other words, only $56.6 \%$ of the process was considered value-added to the customer so that the laboratory can increased their value-added percent by eliminating or reducing the waste in the process.

The team observed technologist's walk pattern depicted in figure 8, the non-value added activities were recognized from the movement of laboratory operators where unnecessary movement observed due to poor organization of space and design of laboratory.

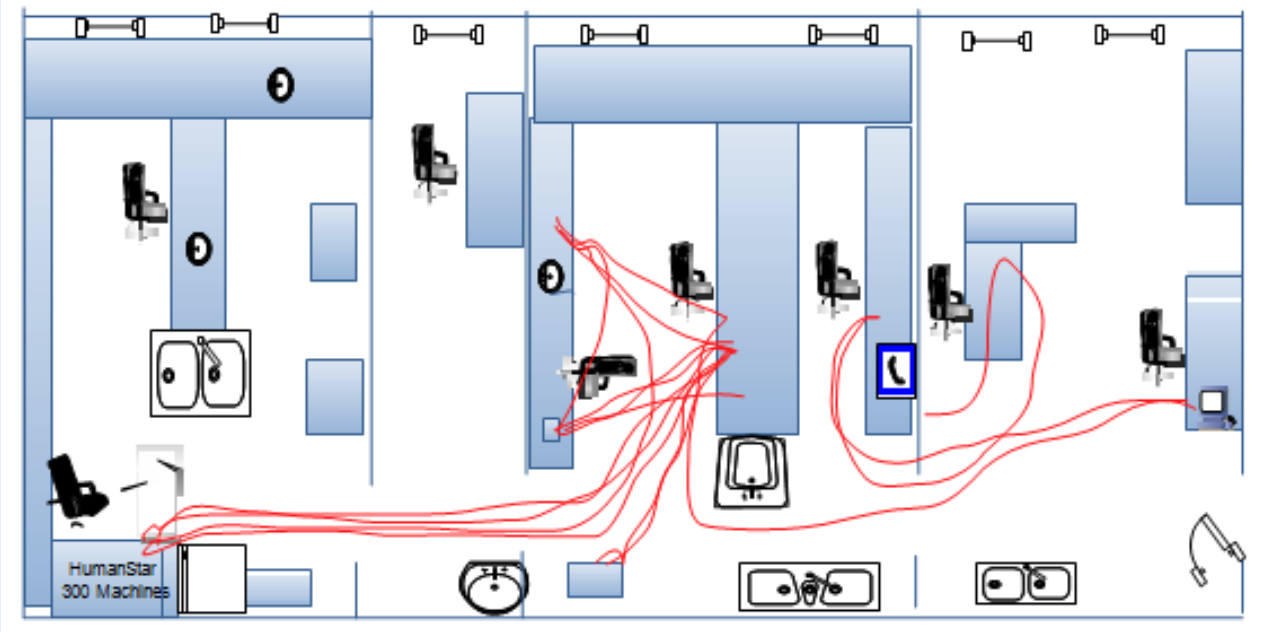

Figure 8: Spaghetti Diagram-Chemistry Blood Sample Analysis-“As Is”, Addis Ababa, Ethiopia.

The operator's movements every day were the same; the study acknowledged there was a room for improvement by modifying the workstation by removing the bench that attached with wall (Room 2). Therefore, it would be sure that the movement of operator can be reduced significantly into sex steps as compared 17 steps of movement that was recorded on existed workflow and twelve steps movements were considered as wastes. The proposed new spaghetti diagram was presented on figure 9.

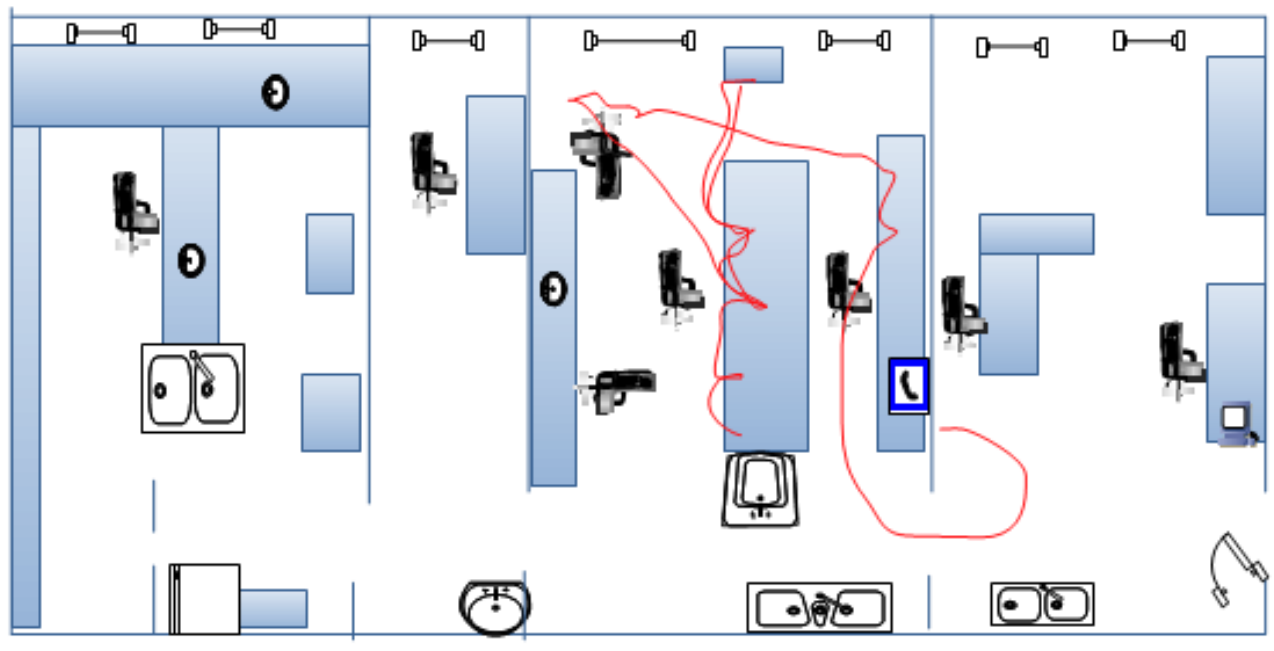

Figure 9: Spaghetti Diagram-Chemistry Lab Testing-“To Be”, Addis Ababa, Ethiopia. 


\section{Discussion}

A total of 649.2 minutes were recognized as non-value added time from 708 samples from current specimen collection workflow. Similar study done in New Jersey, US hospital, BD diagnostic service, the current state consisted of 19 steps, five of which were at risk for specimen identification errors, and three of which were causing unnecessary delays in the specimen collection workflow. The value stream mapping showed that the total valueadded time per day (based on 616 blood specimen collections/day) was 2264.3 minutes, while the non value-added time was 1018.5 minutes [6,7]. This could explain as no quality management system implemented and customer based service provided.

In our study standing blood samples for long period of time until enough number of samples aggregated were documented. As example blood collected for hematological test was left on mixer extra mixing procedure in range of 2.3 minutes to 1.05 hours. According to the clinical and laboratory standard institute (CLSI) recommendation that primary tubes that contain additive should be gently mixed 5 to 10 times (less than 30 seconds) to allow a complete interaction between blood and anticoagulant [8]. Therefore, the effect of prolonged mixing of hematological test can be studied and used for improvement.

The study confirmed that at the pick time of a day the reception should deployed more staff and experienced phlebotomist should be selected for effective time management, and producing reliable and accurate test result since prolonged blood drawing process had direct effect on test measurement. Therefore, the laboratory shall scheduled ahead of time who is going to collect samples since as observed and recognized there were some student in practices or other unqualified personnel involved in the blood drawing process which greatly affect the test result, workflow and turnaround time.

Patients were waited on average between 35 minutes and 2.28 hours. Even if the reception usually opened between 8:20 to 8:30AM, the opening hour of reception in every day was inconsistent. The blood drawing station was three and they were small in size, poor ergonomic workstation used, physical layout and space was not organized. In other study stated that their outpatient phlebotomy clinic was small in space size but had five drawing stations and patients arrive without appointments are seen on a first-come, first-served basis.
Wait times were inconsistent; some patients were seen almost on arrival, but others waited 30 to 40 minutes, or longer. The analysis found an imbalance between demand and staffing, especially early in the day. Patients arriving before the actual opening time caused a backlog that had a ripple effect lasting into the morning. To address that, the team decided to open the centre 30 minutes earlier and increase staffing during the morning hours [9].

This study revealed that the flow coordinator coordinates the process based on patient arrival time (first-come, first serve), and patients who had urine and stool were provide their samples after blood drawing completed. However, study done in Brigham and women's hospital shown that those patients need urine/stool testing provided their specimens collected in the interval between arrival and blood draws [10].

The following were recognized as area of improvement on samples collection workflow such as opening the reception before 30 minutes; assigned flow coordinator everyday; develop a ways for phlebotomist in reduce non-blood drawing activity; use barcode label system, use unique laboratory ID number, make available all collection kits prior collection start; reconfiguring blood drawing station, use ergonomically phlebotomy workstation, change the physical layout of reception, etc. Study done in Brigham and women's hospital, shown that the following opportunities were identified during the pre-lean period: non-blood-drawing activities decreased the amount of time phlebotomists could devote to their patients, patients often arrived before the opening of the phlebotomy station, creating a backlog at the beginning of the day that increased wait time, because patients were not aware of their "place in line," staff sometimes had difficulty identifying and finding the next patient when it was his or her turn [10].

Our finding confirmed that the average patient waiting time after the opening of reception and prior to blood drawing station was 6.06 minutes and patient waiting time in term of pick hour of a day (usually in the morninghigh number of samples) and in the middle of day (less number of samples) were 14.56 and 2.1 minutes respectively. Other study explained that an average patient wait time during a high volume period was 21 minutes. Collecting urine specimens before the blood draw helped decrease wait time. For patients requiring urine collection, the wait time was reduced by $20 \%$. The percentage of time spent performing non-value-added 


\section{Ergonomics International Journal}

tasks decreased, which also contributed to shorter wait times [6].

In this study the average cycle time of blood drawing process was 3.58 and this cycle time were highly depend on how experienced phlebotomist at stage, effective coordination of workflow and number of staff available at pick hour, not necessary depend on number of patients present at time. By modifying the existed workflow using lean principle and creating good ergonomic workstation together with modifying the physical layout of reception, the process cycle efficiency can be increased more than $56.6 \%$. Hence, similar study done Brigham and women's hospital revealed that as cycle times are reduced, productivity increases proportionally and $50 \%$ reduction in cycle time [10].

Our assessment revealed that an average of 2.54, 3.39, and 4.3 minutes was spent delivery of samples to serology, chemistry, and haematology laboratory unit respectively. This was due to proximity problem and understanding their functional relationship between them [9].

Only from chemistry workflow a total of 1231 minutes were identified as non-value added (waste) time and $86.7 \%$ of the time was explained on post-analytical phases. Therefore the study identified that the major waste was from post-and pre-analytical process and by modifying the workflow and physical layout of laboratory it's sure that those waste can be reduced and increase productivity and reduce unnecessary.

To improve the current workflow of specimen collection and chemistry laboratory; to reduce the cycle time and to increase the productivity, the study strongly suggest that laboratory should applied quality management system and look the new quality improvement model like lean model to achieve the customer need. Several medical centers are already publishing their successes with the institution of quality improvements in patient care using such techniques. Likewise, clinical laboratories and consulting industries are migrating to improve quality in an increasingly complex system and the same time reduce cost [11].

\section{Conclusion}

Hospital outpatient reception should be opened earlier at least 30 minutes prior to official opening hour to prevent backlog and add additional staff to reception during pick hour of a day. Assigned a person as workflow coordinator and provide him/her training on it. Use the new business improvement tools like lean in laboratory to reduce waste of unnecessary movement, paper works, etc. Eliminate any non-value activities or process; work only on value-added process to lead laboratory industry and satisfy the customer through re-design the processes again and again. Redesigning of workflow need adoption of modern technology and equipment like using vacationer collection device and its associated tubes rather than syringe needle.

\section{References}

1. Heather WH, Jamie WG, Mindy F, Deanna S, Susan S, et al. (2006) Implementation of Systems Redesign: Approaches to Spread and Sustain Adoption.

2. Tapping D (2006) Value Stream Management for the Lean Office 2003: Lean and Six Sigma: Not for Amateurs. Lab Medicine 37: 78-83.

3. Christopher JB (2008) Using ergonomic principles in the clinical laboratory: Laboratory design. Journal of Clinical leadership and management review.

4. Stankovic AK, DeLaurso E (2010) Quality improvements in the preanalytical phases: Focus on urine specimen workflow. MLO Med Lab Obs 42(3): 20-27.

5. (2006) Stanford Laboratory Standard and Design Guide: Section 1, Version 2.0/11.

6. Stacy FM, Ellen MG, Margaret ML, Jonathan MB, Jose DP, et al. (2009) Applying Lean/Totota production system principles to improve phlebotomy patient satisfaction and workflow. Am J clini pathol 132(6): 914-919.

7. Ana KS (2008) Developing a lean consciousness for the clinical laboratory. JMB 27(3): 354-359.

8. Hoskins D, Erickson J (1998) Laboratory ergonomics, the wake-up call: A case study. Chemical Health and Safety 5(1): 20-23.

9. Brigham and Women's Hospital (2010) Lean tools in the clinical laboratory.

10. Sheryl W (2004) A case study: Clinical Laboratory improves turnaround time. 
11. Joe RM, Joanne S (2010) Application of the Toyota production system improves core laboratory

operations. Am J Clin Pathol 133(1): 24-31.

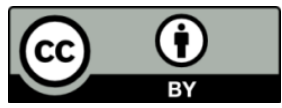

